
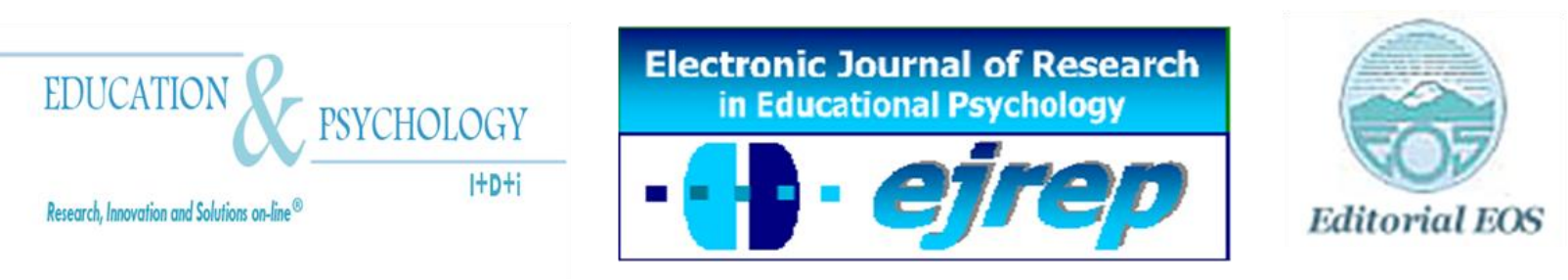

\title{
La Inteligencia Emocional y su Relación con el Género, el Rendimiento Académico y la Capacidad Intelectual del Alumnado Universitario
}

\author{
María de los Dolores Valadez Sierra ${ }^{1}$, \\ María África Borges del Rosal', \\ Norma Ruvalcaba Romero ${ }^{3}$, Karina Villegas ${ }^{1}$ \\ y Maryurena Lorenzo ${ }^{2}$
}

${ }^{1}$ Departamento de Psicología Aplicada, Centro Universitario Ciencias de la Salud, Universidad de Guadalajara, Guadalajara, México

2 Departamento de Psicobiología y Metodología de las Ciencias del Comportamiento, Universidad de La Laguna, Tenerife, España

${ }^{3}$ Departamento de Salud Mental, Centro Universitario Ciencias de la Salud, Universidad de Guadalajara, Guadalajara, México

\section{México / España}

Correspondencia: María de los Dolores Valadez Sierra. Sierra Nevada 950, Col. Independencia C.P. 44100. Guadalajara, Jalisco, México. E-mail: doloresvaladez@yahoo.com.mx

C) Education \& Psychology I+D+i and Editorial EOS (Spain) 


\section{Resumen}

Introducción. La inteligencia emocional se ha relacionado con diversas variables, como el género y el rendimiento académico. En el ámbito de la capacidad intelectual, la literatura muestra controversia, sin que exista un resultado unánime sobre la relación que existe entre estas variables En el presente estudio se analiza el efecto modulador que tiene el rendimiento académico, en una muestra de estudiantes universitarios recién ingresados, comparando por grupos, uno con inteligencia promedio y otro con inteligencia superior, y el género.

Método. La muestra estuvo constituida por 129 estudiantes que ingresaron al centro universitario de ciencias de la salud en el curso escolar 2011-2012, de los cuales 64 presentaban inteligencia superior y 65 promedio. Para medir la inteligencia emocional se utilizó el Mayer, Salovey and Caruso Emotional Intelligence Test (MSCEIT), el test de Matrices Progresivas Escala Avanzada para la medida de la inteligencia y como indicador de rendimiento académico, el promedio de calificaciones.

Resultados. Los resultados muestran que el rendimiento juega un papel diferencial en algunas de las ramas apresadas por el instrumento. Igualmente, aparecen diferencias entre los grupos en Facilitación emocional, Inteligencia Emocional Estratégica y en Comprensión Emocional, siendo en todos los casos mayor la puntuación de las mujeres y de los más inteligentes.

Discusión. Los resultados señalan relación entre inteligencia emocional y rendimiento, si bien difieren, en función del indicador de rendimiento que se tome. Además, se comprueba una relación entre inteligencia emocional, género y capacidad intelectual, apoyando los resultados parcialmente la hipótesis de que las mujeres y las personas con mayor capacidad intelectual presentan más inteligencia emocional.

Palabras Clave: Inteligencia emocional, capacidad intelectual, rendimiento académico, universitarios. 


\title{
Emotional Intelligence and its Relationship with Gender, Academic Performance and Intellectual Abilities of Undergraduates
}

\begin{abstract}
Introduction. Emotional intelligence has been linked to several variables, such as gender, and academic performance. In the area of high intellectual abilities, the literature shows controversy, without a unanimous result on the relationship between both variables. In the present study we analyzed the modulatory effect has academic performance in a sample of college students, one group with normal intelligence and other one with superior intelligence, and gender.
\end{abstract}

Method. The sample consisted of 129 students who admitted in health sciences careers, 64 students had high intelligence and 65 students had normal intelligence. To measure emotional intelligence was used Mayer, Salovey and Caruso Emotional Intelligence Test (MSCEIT) and grade point average for academic performance.

Results. The results show that the performance differential plays a role in some of the branches assessed by the instrument. Similarly, there are differences between the groups in emotional Facilitation, Strategic Emotional Intelligence and Emotional Understanding, being in all cases higher the score in the more intelligent and woman.

Discussion. The results indicate relationship between emotional intelligence and performance, although it differs depending on the performance indicator is taken. In addition, we note a relationship between emotional intelligence and intellectual capacity, the results partially support the hypothesis that women and the most able have more emotional intelligence.

Keywords: Emotional intelligence, high capacity, academic performance, undergraduates.

Received: 04/24/13 Initial acceptance: 06/16/13 Final acceptance: 07/23/13 


\section{Introducción}

La inteligencia emocional (IE) es un tema que ha ido ganando relevancia a partir de los trabajos de Salovey y Mayer (1990), una vez popularizado el término por Goleman (1995), si bien este concepto alude a concepciones distintas e incorpora componentes diferentes. Brackett y Mayer (2003) distinguen dos tipos de modelos: el mixto, que incluye los de la Bar-On (1997) o Schutte et al. (1998), y el de habilidad mental (Brackett y Mayer, 2003), correspondiente al de Mayer y Salovey, que puede considerarse una forma de medida y de análisis más riguroso (Valls, 2007). En un reciente estudio realizado con académicos y profesionales de la Psicología española, señalaron que este modelo era el más conocido y aceptado (Lorenzo, 2012).

Mayer y Salovey (1997) proponen un modelo jerárquico, conocido como el Four Branches Model (Ruvalcaba y Gallegos, en prensa), compuesto por cuatro habilidades: a) para percibir y valorar con exactitud la emoción, b) para acceder y/o generar sentimientos cuando éstos facilitan el pensamiento, c) para comprender la emoción y el conocimiento emocional y d) para regular las emociones que promueven el crecimiento emocional e intelectual. Los autores afirman que estas cuatro habilidades o ramas están ordenadas, dándose una progresión creciente en su adquisición (Mayer, Salovey y Caruso, 2004). El enfoque de habilidad, al poder ésta adquirirse, permite ser aprendida y enseñada, permitiendo también su aplicación en el ámbito educativo, para la mejora del bienestar y la calidad de vida de las personas (Ruvalcaba y Gallegos, en prensa).

La inteligencia emocional se ha relacionado con el bienestar subjetivo (Bar-On, 2005), la felicidad (Bar-On, 2010), la salud mental (Bhullar, Shutte y Malouff, 2012; Martínez, Piqueras y Ramos, 2010; ), la prevención de conductas de riesgo (Brackett y Mayer, 2003), la competencia social (Brasseur, y Gregoire, 2011; Zavala, Valadez y Vargas, 2008;), la robustez de las relaciones familiares (Chan, 2005), así como con locus de control y autoeficacia (Bellamy, Gore y Sturgis, 2005) y el ajuste socioescolar de los alumnos (Pena y Repetto, 2008).

Un tema controvertido es la relación entre IE y género, ya que diversos estudios han encontrado diferencias en función del género, que favorecen, en general, a las mujeres (Extremera, Duran y Rey, 2007; Extremera, Fernández-Berrocal y Salovey, 2006; Valadez, Pérez y 
Beltrán, 2010; Thompson, Waltz, Croyle y Pepper, 2007), o bien lo hacen de forma diferencial: las mujeres puntúan más en aspectos que tienen que ver con la relación con otros, mientras que los varones lo hacen en la resolución de problemas (Sánchez, Fernández-Berrocal, Montañes y Latorre, 2008). No obstante, los resultados no son unánimes, dándose ausencia de diferencias (Chan, 2005; Prieto-Rincón, Inciarte-Mundo, Rincón-Prieto y Bonilla, 2008), o encontrando variables moduladoras, como la edad (Cabello, Fernández-Berrocal, Castillo y Extremera, 2011), las pautas de socialización de los padres (Sánchez, Rodríguez y Padilla, 2007), o el locus de control y la autoeficacia (Bellamy et al., 2005).

En el rendimiento escolar, uno de los aspectos más estudiados, los hallazgos obtenidos están lejos de ser concluyentes. Por una parte, algunos estudios muestran relación entre IE y rendimiento (Bar-On, 2007; Extremera y Fernández-Berrocal, 2009; Gil-Olarte, Palomera y Brackett, 2006; Jiménez y López, 2013; Lee y Olszewski-Kubilius, 2006; Woitaszewski y Aalsma, 2004; Pérez-Pérez y Castejón, 2006) o bien se observa que tiene un papel mediador en el éxito académico (MacCann, Fogarty y Zeidner, 2011). En otros casos se muestra como un adecuado predictor del rendimiento académico (López, Mestre y Abellán, 2011; Parker, Summerfeldt, Hogan, y Majeski, 2004) o bien se da relación significativa, pero con un escaso tamaño del efecto (Mayer et al., 2004).

Otros estudios, no obstante, no apoyan de forma clara la relación entre rendimiento e IE. En el trabajo de Sánchez et al., (2007) la relación aparece en un solo factor, de forma que mayor atención emocional conlleva menor rendimiento académico. En otros casos se observa relación cuando se controlan variables cognitivas y de personalidad (Barchard, 2003) o bien lo contrario: la significación de la correlación desaparece controlando la inteligencia (Brackett y Salovey, 2006). Newsome, Day y Catano (2000) encontraron ausencia de relación positiva entre inteligencia emocional, medida con el EQ-i, y el rendimiento académico en universitarios canadienses.

En el área de la capacidad intelectual, se han encontrado relaciones entre inteligencia cognitiva y emocional (Gil et al., 2006). Cuando los estudios se centran en personas con alta capacidad intelectual, la relación con aspectos tales como las relaciones interpersonales y el ajuste personal y social ha sido un tema de debate secular, que sigue abierto, si bien últimas investigaciones señalan ausencia de diferencias en estos aspectos con respecto a grupos normativos (Borges, Hernández-Jorge y Rodríguez-Naveiras, 2011; López y Sotillo, 2009). En 
cuanto a diferencias en inteligencia emocional entre grupos de diferente capacidad intelectual, Brasseur, y Gregoire (2011) no encontraron diferencias entre superdotados y adolescentes de inteligencia normal en inteligencia emocional, mientras que tanto Prieto et al. (2008) como Valadez et al. (2010) muestran que los superdotados tienen mayor competencia en inteligencia emocional.

En resumen, la inteligencia emocional es un rótulo tremendamente prometedor, pero que precisa rigurosa y amplia investigación para esclarecer el papel que juega en áreas donde se postula que resulta relevante. Los resultados poco concluyentes es posible que se deban a la poca homogeneidad del concepto de inteligencia emocional o a la forma de medirlo. Los trabajos de Valadez et al. (2010) y de Zeidner, Shani-Zinovich y Matthews (2005), de forma nada sorprendente, muestran que lo hallazgos dependen del tipo de instrumento usado para la medida de la inteligencia emocional. Por todo ello, resulta de gran importancia la investigación en inteligencia emocional y los factores relacionados.

\section{Objetivo e hipótesis}

El objetivo de este trabajo es determinar la relación existente entre capacidad intelectual e inteligencia emocional, tomando en cuenta el género y el rendimiento académico. En este sentido, se plantean tres hipótesis: a) la inteligencia emocional se relaciona con el rendimiento académico, b) los más inteligentes presentan mayor inteligencia emocional, y c) las mujeres, independientemente de su capacidad intelectual, presentan una mayor inteligencia emocional.

\section{Método}

\section{Participantes}

Tomaron parte en el estudio 129 estudiantes, que ingresaron a las diferentes carreras del Centro Universitario de Ciencias de la Salud en el curso escolar 2011-12, con un rango de edad entre 17 y 20 años (Edad media 18,26, SD=0,6), de los cuales 49 eran varones y 80 mujeres, cursando las carreras del área de conocimiento de Ciencias de la Salud: Cirujano Dentista (14), Enfermería (14), Medicina (63), Nutrición (12) y Psicología (26).

Se establecieron dos grupos en función de las puntuaciones obtenidas en el Test de Matrices Progresivas, Escala Avanzada, el primero formado por estudiantes de inteligencia 
superior, que alcanzaron un percentil de 90 o superior en dicha prueba $(n=64)$, y un grupo inteligencia promedio, cuya puntuación correspondía con un percentil de 50 (n=65).

\section{Instrumentos}

Test de Matrices Progresivas Escala Avanzada (Raven, Cort, y Raven, 2001). Los test de Matrices progresivas son un test de capacidad intelectual y están considerados "libre de cultura”. La escala Avanzada mide la eficiencia intelectual, permitiendo diferenciar a personas con una aptitud superior. Está conformado por dos cuadernos separados, Serie I y Serie II. La Serie I consta de 12 items, generalmente se usa para que el evaluado se familiarice con la naturaleza de los problemas que se le presentarán en la Serie II. La Serie II consta de 36 ítems de dificultad creciente, en cada ítem se debe elegir entre 8 opciones, la figura correcta que complete la matriz.

Los autores (Raven et. al., 2001) informan una fiabilidad test-retest de 0,91, para el caso de adultos Británicos de capacidad intelectual superior al promedio. Para la validez de constructo, en el análisis factorial, se obtuvo una saturación de "g" de 0,82. En México, en el estudio efectuado por Méndez-Sánchez y Palacios-Salas (2001), para obtener los baremos en la Cd. de Aguascalientes, encontraron puntuaciones similares a las de Raven et. al. (2001). El test se puede aplicar en forma individual o colectiva y con o sin límite de tiempo. En este estudio se aplicó la serie II de forma grupal y sin límite de tiempo.

\section{Adaptación Española del Test de Inteligencia Emocional Mayer-Salovey-Caruso} (MSCEIT) (Extremera y Fernández-Berrocal, 2009). Evalúa la inteligencia emocional midiendo la ejecución de las personas en tareas y resolución de problemas emocionales en dos áreas: Inteligencia Experiencial e Inteligencia Emocional Estratégica, que se despliegan en cuatro ramas: a) percepción emocional; b) facilitación emocional; c) comprensión emocional; y d) manejo emocional. Cada rama tiene asociadas dos tareas: Caras y Dibujos, Facilitación y Sensaciones, Cambios y Combinaciones, Manejo Emocional y Relaciones Emocionales, respectivamente.

La versión española tiene una fiabilidad en la puntuación total de 0,95 y en las áreas experiencial y estratégica, de 0,93 y de 0,90 respectivamente, todas similares a la versión original. La validez factorial obtenida en la adaptación al castellano del MSCEIT coincide con la 
estructura del test original, obteniendo un modelo de cuatro factores acorde a la teoría de Mayer y Salovey.

\section{Variables criteriales}

Promedio de preparatoria: Calificación promedio obtenida al egresar del Bachillerato.

Prueba de Aptitud Académica $(P A A)^{1}$ : Prueba a la que se somete al estudiantado mexicano para ingresar a una de las carreras que oferta la universidad, midiendo tres áreas cognoscitivas: razonamiento lógico matemático; razonamiento verbal y lectura crítica y redacción.

Puntos de admisión: Promedio entre la calificación obtenida como promedio en preparatoria y la prueba de aptitud.

Promedio actual: Es el promedio de calificaciones que el alumnado alcanza en este ciclo escolar.

\section{Procedimiento}

El jefe del departamento de psicología básica solicitó permiso a los profesores que imparten docencia en las carreras mencionadas para evaluar a los alumnos. Obtenido el consentimiento del profesorado, se explicó al alumnado en que consistían los instrumentos a aplicar y se les invitó a participar de forma voluntaria. La aplicación de las pruebas fue colectiva.

\section{Diseño y análisis de datos}

El diseño fue ex post facto retrospectivo, de comparación de grupos de casos y controles. La relación el rendimiento académico y las puntuaciones en las escalas del MSCEIT se analizó mediante el coeficiente de correlación de Pearson. La determinación de diferencias debidas a capacidad y género mediante un análisis de covarianza, ANCOVA, de dos factores, controlando el efecto del rendimiento académico al utilizar como covariante la Prueba de Aptitud.

\section{Resultados}

Se analizaron las relaciones entre las distintas puntuaciones alcanzadas en las escalas

\footnotetext{
${ }^{1}$ http://escolar.udg.mx/sites/default/files/Guia\%20de\%20estudios\%20PAA.pdf
} 
que componen el MSCEIT y las medidas criteriales de rendimiento (véase tabla número 1). Como se puede observar, las calificaciones obtenidas durante el curso (promedio actual) resultan independientes de las medidas de inteligencia emocional. La variable criterial más relacionada es la Prueba de Aptitud Académica, que a su vez coincide en ser la que tiene un mayor componente cognitivo y de conocimientos adquiridos en los estudios de bachillerato, alcanzando correlaciones significativas con el Cociente de Inteligencia Emocional, con el Área de Inteligencia Emocional Estratégica y en una de sus ramas, Comprensión Emocional, y en las tareas correspondiente. Sorprendentemente, la variable Puntos de Admisión alcanza correlaciones significativas en las mismas escalas que la Prueba de Aptitud. La tarea Manejo de Emociones, en cambio, resulta significativa con el Promedio de Preparatoria y en Puntos de Admisión.

Tabla 1. Coeficientes de correlación de Pearson entre escalas del MSCEIT y las medidas criteriales de rendimiento

\begin{tabular}{lcccc}
\hline & $\begin{array}{c}\text { Promedio } \\
\text { Preparatoria }\end{array}$ & PAA & $\begin{array}{c}\text { Puntos de } \\
\text { admisión }\end{array}$ & $\begin{array}{c}\text { Promedio } \\
\text { actual }\end{array}$ \\
\hline Cociente I.E. &, 035 &, $201^{*}$ &, 177 &,- 035 \\
I.E. Experiencial &,- 035 &, 140 &, 097 &,- 134 \\
Percepción emocional &,- 041 &, 042 &, 015 &,- 088 \\
$\quad$ Caras &,- 010 &,- 016 &,- 017 &,- 012 \\
$\quad$ Dibujos &,- 045 &, 062 &, 030 &,- 094 \\
Facilitación emocional &, 000 &, 133 &, 106 &,- 106 \\
$\quad$ Facilitación &,- 009 &, 143 &, 111 &,- 098 \\
$\quad$ Sensaciones &, 081 &, 044 &, 071 &,- 015 \\
I.E. Estratégica &, 072 &, $193^{*}$ &, $186^{*}$ &, 061 \\
Comprensión emocional &, 093 &, $314 * *$ &, $293 * *$ &, 119 \\
$\quad$ Cambios &, 151 &, $253 * *$ &, $269 * *$ &, 109 \\
Combinaciones &,- 043 &, $290^{* *}$ &, $214 *$ &, 057 \\
Manejo emocional &, 115 &, 110 &, 139 &, 082 \\
$\quad$ Manejo emocional &, $192 *$ &, 175 &, $225^{*}$ &, 091 \\
Relaciones emocionales &,- 040 &,- 014 &,- 029 &, 042 \\
\hline
\end{tabular}


Para la determinación de las diferencias entre estudiantado de inteligencia normal y de inteligencia superior en las diversas escalas de Inteligencia Emocional se calculó un análisis de covarianza de dos factores, género y capacidad, tomando como covariante la Prueba de Aptitud Académica. En la tabla número 2 se presentan las medias y desviaciones típicas en cada escala del MSCEIT en función de la capacidad y el género.

Tabla 2. Medias y desviaciones típicas de las escalas de I.E. por genero

\begin{tabular}{lcccc}
\hline Escalas & \multicolumn{2}{c}{ Inteligencia superior } & \multicolumn{2}{c}{ Inteligencia promedio } \\
\hline & Hombres & Mujeres & Hombres & Mujeres \\
I.E. & $105,54(11,6)$ & $107,89(8,4)$ & $98,28(11,6)$ & $103,5(10,7)$ \\
I.E. Experiencial & $102,50(12,0)$ & $104,18(9,2)$ & $96,36(12,3)$ & $99,84(18,9)$ \\
Percepción emocional & $106,08(12,7)$ & $107,87(10,2)$ & $102,84(14,5)$ & $107,05(12,5)$ \\
$\quad$ Caras & $102,21(12,9)$ & $108,21(8,9)$ & $100,12(13,3)$ & $106,34(9,9)$ \\
$\quad$ Dibujos & $106,58(12,3)$ & $104,95(10,0)$ & $102,92(13,8)$ & $104,97(13,4)$ \\
Facilitación emocional & $97,00(9,8)$ & $98,74(10,3)$ & $90,56(9,9)$ & $96,53(11,6)$ \\
$\quad$ Facilitación & $93,42(10,9)$ & $95,21(11,4)$ & $87,00(10,2)$ & $92,05(12,9)$ \\
$\quad$ Sensaciones & $102,58(10,8)$ & $103,82(10,8)$ & $98,68(11,1)$ & $104,63(11,3)$ \\
I.E. Estratégica & $107,88(11,8)$ & $109,42(8,6)$ & $101,08(11,8)$ & $101,24(18,0)$ \\
Comprensión Emocional & $110,92(10,3)$ & $111,71(9,9)$ & $105,40(13,2)$ & $105,16(10,7)$ \\
$\quad$ Cambios & $109,21(10,7)$ & $111,45(9,7)$ & $104,80(11,9)$ & $105,82(13,3)$ \\
Combinaciones & $110,79(12,0)$ & $107,95(10,1)$ & $104,52(15,6)$ & $103,16(9,0)$ \\
& $102,75(12,9)$ & $104,61(8,8)$ & $97,44(11,5)$ & $101,92(11,6)$ \\
Relaciones & $103,38(9,1)$ & $106,26(11,0)$ & $100,08(9,1)$ & $104,37(10,2)$ \\
\hline Manejo Emocional & $101,38(11,3)$ & $102,26(9,1)$ & $95,24(10,1)$ & $99,11(12,6)$ \\
Manejo & & &
\end{tabular}

En todos los casos que existen diferencias significativas, las puntuaciones más altas corresponden a las mujeres, en la variable género, y a los estudiantes más inteligentes en la variable capacidad.

En el Cociente de Inteligencia Emocional la covariante es significativa, $F(1,117)=$ $5,37, p=0,022, \eta^{2} \mathrm{p}=0,044$, lo que señala que no existe efecto debido a la prueba de aptitud. Se comprueba que los más inteligentes puntúan más alto, $F(1,117)=6,24, p=0,014 \eta^{2} \mathrm{p}=$ 0,051 así como las mujeres $F(1,117)=6,18, p=0,014, \eta^{2} \mathrm{p}=0,050$. 
En el área de Inteligencia Emocional Experiencial y en una de sus ramas, Percepción Emocional, no se dan diferencias significativas, así como en una de sus tareas, Dibujo. En la otra tarea, Caras, las mujeres puntúan significativamente más alto, $F(1,117)=8,20, p=$ 0,005, siendo el tamaño del efecto $\eta^{2} \mathrm{p}=0,066$, si bien hay que tomar estos datos con cautela, ya que los grupos son heterocedásticos, $F(3,18)=2,91, p=0,038$. En la otra rama, Facilitación emocional, igualmente las mujeres obtienen puntuaciones superiores, $F(1,117)=4,58$, $p=0,03, \eta^{2} p=0,038, \mathrm{y}$ también lo hacen los más inteligentes, $F(1,117)=4,06, p=0,046, \eta^{2} p$ $=0,034$. En cuanto a las tareas asociadas, no se encuentran diferencias ni en Facilitación ni en Sensaciones.

En Inteligencia Emocional Estratégica hay efectos debidos a la variable capacidad, $F$ $(1,129)=7,16, p<0,01, \eta^{2} p=0,058$, a favor de los estudiantes más inteligentes. En la rama asociada, Comprensión Emocional, se controla el efecto de la prueba de aptitud, $F(1,117)=$ 10,80, $p=0,001, \eta^{2} \mathrm{p}=0,085$, y se encuentran diferencias a favor de los más inteligentes, $\mathrm{F}$ $(1,117)=5,82, \mathrm{p}<0,01, \eta^{2} p=0,047$. En las tareas asociadas, mientras en Cambio, controlada la prueba de aptitud, $F(1,117)=7,61, p<0,010, \eta^{2} p=0,061$, no aparecen diferencias ni debidas a género ni a capacidad, en Combinaciones, controlado el efecto de la covariante, $F$ $(1,117)=7,506, p^{<} 0,01, \eta^{2} p=0,060$, hay diferencias debidas a la capacidad, $F(1,117)=$ 4,84, $p<0,05, \eta^{2} p=0,040$, debiendo, como en el caso de Caras, considerar este resultado con cuidado, debido a mostrar heterocedasticidad, $(\mathrm{F}(3,18)=4,05, p<0,01)$. No hay diferencias en la rama de Manejo Emocional ni en las tareas relacionadas (Manejo emocional y Relaciones emocionales).

\section{Discusión y conclusiones}

Los resultados aquí obtenidos ponen de manifiesto la existencia de relación entre inteligencia emocional y rendimiento, si bien no es consistente y está en función del indicador de rendimiento que se considere. Las puntuaciones obtenidas en el curso que están realizando no resultan significativas, y prácticamente tampoco la media de rendimiento con respecto a la calificación obtenida en el Promedio en Preparatoria. En esta puntuación criterial influyen otras habilidades además de los conocimientos (actitud, limpieza en la presentación de tareas y actividades), siendo así las calificaciones un constructo multidimensional, conformado por una variedad de factores, que pueden ser incluso ajenos al alumnado (Ruvalcaba y Gallegos, 
en prensa) lo que pone de manifiesto que la inteligencia emocional medida a través del MSCEIT tiene más peso en factores cognitivos.

Las relaciones obtenidas con la Prueba de Aptitud Académica confirman este aserto, pues de los cuatro indicadores de rendimiento es el que presenta correlaciones más relevantes, pero solo en una de las ramas de Inteligencia Emocional Estratégica, la de Comprensión Emocional, lo que se explica al ser precisamente esta área la que está vinculada principalmente con la cognitiva, es decir la capacidad para etiquetar las emociones, comprender su significado, sus causas y la transición entre las mismas. Por tanto, la primera hipótesis planteada queda corroborada sólo en parte, y resulta coincidente con lo observado en diferentes investigaciones (Bar-On, 2007; Extremera y Fernández-Berrocal, 2009; Gil-Olarte et al., 2006; Jiménez y López, 2013; Lee y Olszewski-Kubilius, 2006; Woitaszewski y Aalsma, 2004; Pérez-Pérez y Castejón, 2006). Sin embargo, se pone de manifiesto que el rótulo "inteligencia emocional", tal como lo apresa el MSCEIT, es un constructo complejo, y su relación con rendimiento académico debe establecerse de forma clara y analizando otras variables que puedan ayudar a explicarla.

La variable Puntos de Admisión, en la medida que está formada por las dos primeras, la Prueba de Aptitud Académica y el Promedio actual, presenta correlaciones significativas donde las variables de partida lo hacen. La relación encontrada a nivel relacional entre inteligencia emocional y rendimiento pone de manifiesto el interés de emplear dicho indicador como covariante. Así, juega un papel relevante en las tareas relacionadas con comprensión emocional, de tal forma que en Cambios las diferencias del efecto de capacidad están producidas por el rendimiento académico.

Los resultados que aquí se presentan permiten corroborar la existencia de diferencias debidas al género en inteligencia emocional, corroborando la tercera hipótesis planteada. Las puntuaciones obtenidas en todas las escalas favorecen a las mujeres, resultando significativa la diferencia entre ambos sexos en el Cociente de Inteligencia Emocional, lo cual es concordante con los resultados informados en diversos estudios (Extremera et al., 2007; Extremera et al., 2006; Valadez et al., 2010; Thompson, Waltz, Croyle y Pepper, 2007), si cuando se analizan las subsiguientes puntuaciones las diferencias son significativas solo en Facilitación Emocional y en la tarea de Caras, lo que pone de manifiesto que es preciso incluir variables moduladoras que permitan entender la relación entre inteligencia emocional y género. 
Frente a otros estudios, donde no se obtiene relación entre capacidad intelectual e inteligencia emocional, los presentes resultados apoyan parcialmente la hipótesis de que los más capaces tienen mayor inteligencia emocional, siendo las diferencias siempre a favor de los más inteligentes, confirmando los hallazgos obtenidos por Prieto et al. (2008) y Valadez et al. (2010), si bien la significación de estas diferencias no aparece de forma general en todas las escalas y, en las que se dan, con tamaños del efecto escasos. En la puntuación global de Cociente de Inteligencia General, una vez controlado el influjo del rendimiento, se observa también efecto debido a la capacidad intelectual. En las tareas asociadas no se encuentran diferencias significativas, salvo en Combinaciones. Parece haber diferencias en las estructuras más amplias (Inteligencia Emocional Estratégica, Facilitación Emocional y Comprensión emocional) pero estas diferencias no se mantienen en las tareas, salvo, como hemos dicho, en Combinaciones.

El terreno de la inteligencia emocional sigue exigiendo una investigación seria y rigurosa que establezca que es lo que realmente se incluye bajo el paraguas del rótulo. No sólo habría que intentar separar los diversos modelos que concurren en la actualidad y compiten para defender la denominación, también es importante dilucidar de la forma más clara posible qué variables se relacionan con inteligencia emocional, y cuál es el papel que juegan.

Dada la relevancia que tiene el descubrir qué contribuye y en qué medida al rendimiento académico, es importante tratar de establecer las variables que son posibles predictoras de dicho rendimiento. Pero la misma forma de determinar los resultados de aprendizaje no resulta sencilla, como se ha puesto de manifiesto en el presente trabajo. Queda claro que dependiendo de la forma en que se operativice dicho rendimiento académico pueden darse unos resultados u otros. No obstante, la forma de medirlo queda muy lejos del alcance de esta investigación.

Con respecto a su relación con capacidad intelectual, el problema de la definición del concepto relativo a superdotación, alta capacidad, sobredotación, y demás rótulos relacionados, es también similar al de inteligencia emocional, pues la conceptualización de lo que se entiende por ello pasa por el modelo al que el investigador se adscriba. Por esta razón, también hay dificultades en determinar qué características se asocian a esta capacidad, lo que conlleva además problemas prácticos, como puede ser quién puede ser incluido en el colectivo y, por ello, beneficiarse de las medidas que se implanten, en concreto en el campo educativo 
(Borges, 2011). En el presente estudio, evitando la adscripción a un modelo concreto en referencia a la capacidad intelectual superior, se ha optado por un modelo psicométrico sencillo, considernado inteligencia superior cuando se supera el percentil 90 en la muy utilizada prueba Raven escala Avanzada.

La relación entre inteligencia emocional y capacidad intelectual, como se muestra en este estudio, si bien parece confirmarse en parte, se asocia sólo a unas ramas y, dentro de ellas, sólo a determinadas tareas. Puesto que los defensores del modelo postulan que la adquisición de habilidades se da en un nivel de progresión creciente (Mayer et al., 2004), los resultados aquí obtenidos parecen poner de manifiesto que las capacidades cognitivas tienen relevancia en unos aspectos y no en otros, independientemente de la progresión de su adquisición.

En este estudio se ha circunscrito a una muestra del alumnado de la Universidad de Guadalajara, en concreto a los recién ingresados. Sería conveniente replicar esta investigación en otras muestras de universitarios, así como en grupos de adultos no estudiantes, para analizar y poder generalizar el alcance de la influencia de la inteligencia emocional en la capacidad intelectual. Por otra parte, el cumplimiento parcial de las hipótesis no permite llegar a un posicionamiento claro en el complejo debate de las relaciones entre genéro, rendimiento, inteligencia cognitiva e inteligencia emocional. Parece, por tanto, relevante, como se ha mencionado anteriormente, seguir ahondando en la cuestión e incluir otras variables que permitan ir aclarando estas relaciones, así como la validez que la inteligencia emocional puede tener en la predicción del rendimiento académico, como postula el modelo de Mayer et al. (2004).

\section{Referencias}

Barchard, K. (2003). Does emotional Intelligence assist in the prediction of Academic Success? Educational and Psychological Measurement, 63(5), 840-858. doi: $10.1177 / 0013164403251333$

Bar-On, R. (1997). The Emotional Quotient Inventory (EQ-i): Technical manual. Toronto, Canada: Multi-Health Systems, Inc.

Bar-On, R. (2005). The impact of emotional intelligence on subjective well-being. Perspectives in Education, 23, 41-61.

Bar-On, R. (2007). How important is it to educate people to be emotionally intelligent, and 
can it be done? En Bar-On, R., Maree, J.G. \& Elias, M. J. (Eds.), Educating people to be emotionally intelligent (pp. 1-14). Westport, CT: Praeger.

Bar-On, R. (2010). Emotional intelligence: an integral part of positive psychology. South African Journal of Psychology, 40(1), 54-62. doi: 10.1177/008124631004000106

Bellamy, A., Gore, D. y Sturgis, J. (2005). Examining the relevance of emotional intelligence within educational programs for the gifted and talented. Electronic Journal of Research in Educational Psychology, 3(2), 53-78.

Borges, A. (2011). Problemas conceptuales y metodológicos en altas capacidades intelectuales. Ponencia presentada al Primer Congreso Internacional de Aptitudes Sobresalientes y Talentos. Guadalajara (México).

Borges, A., Hernández-Jorge, C. y Rodríguez-Naveiras, E. (2011). Evidencias contra el mito de la inadaptación de las personas con altas capacidades intelectuales. Psicothema, 23, 362-367.

Brackett, M.A.y Mayer, J.D. (2003). Convergent, discriminant and incremental validity of competing measures of emotional intelligence. Personal and Social Psychological Bulletin, 29, 1147-1158. doi: 10.1177/0146167203254596

Brackett, M.A. y Salovey, P. (2006). Measuring emotional intelligence with the MayerSalovery-Caruso Emotional Intelligence Test (MSCEIT). Psicothema, 18, 34-41.

Bhullar, N., Schutte, N.S. y Malouff, J.M. (2012). Associations of individualisticcollectivistic orientations with emotional intelligence, mental health, and satisfaction with life: a tale of two countries. Individual Differences Research, 10(3), 165-175.

Brasseur, S. y Gregoire, J. (2011). L'intelligence émotionnelle-Trait chez les adolescents à haut potentiel: Spécificités et liens avec la réussite scolaire et les compétences sociales. Enfance, 62(1), 59-76.

Cabello, R., Fernández-Berrocal, P., Castillo, R., Extremera, N. (2011). Are women more emotionally intelligent than men? Empirical evidence. En III International Congress of Emotional Intelligence, Book of Abstracts. Croatia.

Chan, D.W. (2005). Self-perceived creativity, family hardiness, and emotional intelligence of Chinese gifted students in Hong Kong. The Journal of Secondary Gifted Education, XVI (2/3), 47-56. doi: 10.4219/jsge-2005-471

Extremera, N., Durán, A. y Rey, L. (2007). Perceived Emotional Intelligence and dispositional optimism-pessimism: Analyzing their role in predicting psychological adjustment among adolescents. Personality and Individual Differences, 42(6), 1069-1079. doi.org/10.1016/j.paid.2006.09.014 
Extremera, N. y Fernández-Berrocal, P. (2009). Adaptación Española del Test de Inteligencia Emocional Mayer-Salovey-Caruso (MSCEIT). Madrid: TEA Ediciones.

Extremera, N., Fernández-Berrocal, P. y Salovey, P. (2006). Spanish version of the MayerSalovey-Caruso Emotional Intelligence Test (MSCEIT). Version 2.0: Reliabilities, age and gender differences. Psicothema, 18, 42-48.

Gil-Olarte, M., Palomera, R. y Brackett, M. (2006). Relating emotional intelligence to social competence and academic achievement in high school students.Psicothema 18, 118123.

Goleman, D. (1995). Emotional intelligence: Why it can matter more than IQ. New York: Bantam Books.

Jiménez, M.I. y López, E. (2013) Impacto de la Inteligencia Emocional percivida, actitudes sociales y expectativas del profesor en rendimiento académico. Electronic Journal of Research in Educational Psychology, 11(1), 75-98.

Lee, S.Y. y Olszewski-Kubilius, P. (2006). The emotional intelligence, moral judgment, and leadership of academically gifted adolescents. Journal for the Education of the Gifted, 30(1), 29-67.

Lorenzo, M. (2012). Acercamiento metodológico a la opinión de profesionales de la psicología sobre inteligencia emocional. Revista de Investigación y Divulgación en Psicología y Logopedia, 2(2), 28-34.

López Fernández, C., Mestre Navas, J. M. y Abellán Hervás, M. J. (2011). Relación de la inteligencia emocional con el desempeño en los estudiantes de enfermería (Tesis Doctoral, Universidad de Cádiz). Recuperada de: http://serviciopublicaciones.uca.es/uploads/tienda/tesis/6d0a35ce4f7a105e53f7c989e3 89871065bd1f5b.pdf

López, V. y Sotillo, M. (2009). Giftedness and social adjustment: Evidence supporting the resilience approach in Spanish-speaking children and adolescents. High Ability Studies, 20(1), 39-53. doi:10.1080/13598130902860739

MacCann, C., Fogarty, G.J. y Zeidner, M. (2011). Coping mediates the relationship between emotional intelligence (EI) and academic achievement. Contemporary Educational Psychology, 36(1), 60-70. doi.org/10.1016/j.cedpsych.2010.11.002

Martínez, A., Piqueras, J. y Ramos, V. (2010). Inteligencia emocional en la salud física y mental. Electronic Journal of Research in Educational Psychology, 8(2), 1696-2095.

Mayer, J. y Salovey, P. (1997). What is Emotional Intelligence? En Mestre y Fernández- 
Berrocal (2007). Manual de Inteligencia Emocional. Madrid: Pirámide.

Mayer, J., Salovey, P. y Caruso, D. (2004). Emotional Intelligence: Theory, Findings, and Implications. Psychological Inquiry, 15(3), 197-215. doi: 10.1207/s15327965pli1503_02

Mendez-Sánchez, C. y Palacios-Salas, P. (2001). Estandarización del test de Matrices Progresivas de Raven, escala avanzada (MPA). México: Paidós.

Newsome, S., Day, A., y Catano, V. (2000). Assessing the predictive validity of Emotional Intelligence. Personality and Individual Differences, 29, 1005-1016. doi.org/10.1016/S0191-8869(99)00250-0

Parker, J., Summerfeldt, L., Hogan, M. y Majeski, S. (2004). Emotional Intelligence and Academic Success: examining the transition from high school to university. Personality and Individual Differences 36, 163-172. doi.org/10.1016/S0191-8869(03)00076-X

Pena, M. y Repetto, E. (2008). Estado de la investigación en España sobre inteligencia emocional en el ámbito educativo. Revista Electrónica de Investigación Psicoeducativa, 15(6), 400-420.

Pérez-Pérez, N. y Castejón, J. (2006). Relaciones entre la Inteligencia Emocional y el Cociente Intelectual con el Rendimiento Académico en estudiantes Universitarios. Revista Electrónica de Motivación y Emoción IX (22).

Prieto, M.D., Ferrándiz, C., Ferrando, M. Sainz, M., Bermejo, R. y Hernández, D. (2008). Inteligencia emocional en alumnos superdotados: un estudio comparativo entre España e Inglaterra. Revista Electrónica de Investigación Psicoeducativa, 15(6), 297-320.

Prieto-Rincón, D., Inciarte-Mundo, J., Rincón-Prieto, C. y Bonilla, E. (2008). Estudio del coeficiente emocional en estudiantes de medicina. Revista Chilena de NeuroPsiquiatría, 46(1), 10-15. doi.org/10.4067/S0717-92272008000100002

Raven, J.C., Cort, J.H y Raven, J. (2001). Test de Matrices Progresivas.Manual. TEA Ediciones.

Ruvalcaba, N.A y Gallegos, J. (en prensa). Inteligencia emocional percibida y rendimiento académico en estudiantes de bachillerato. En García, M. y Samaniego, R. (Coords) La psicología de la salud y sus campos de aplicación. México: Consorcio de Universidades Mexicanas.

Salovey, P. y Mayer, J. (1990). Emotional Intelligence. Imagination, Cognition and Personality, 9(3), 185-211. doi: 10.2190/DUGG-P24E-52WK-6CDG

Sánchez, M.P., Rodríguez, M.C. y Padilla, V.M. (2007) ¿La inteligencia emocional está relacionada con el rendimiento académico? IPyE: Psicología y Educación, 1(1), 54-66. 
Sánchez, M., Fernández-Berrocal, P. Montañes, J. y Latorre, J. (2008). ¿Es la inteligencia emocional una cuestión de género?, socialización de las competencias emocionales en hombres y mujeres y sus implicaciones. Revista Electrónica de Investigación Psicoeducativa, 15(6), 455-474.

Schutte, N.S. Malouff, J.M., Hall L.E., Haggerty, D.J. Cooper, J.T., Golden, C.J. and Dornheim, L. (1998). Development and validation of a measure of emotional intelligence. Personality and Individual Differences, 25(2), 167-177.

Thompson, B., Waltz, J. Croyle K, y Pepper, A. (2007). Trait Meta Mood and affect as predictors of somatic symptoms and Life Satisfaction. Personality and individual Differences, 43(7), 1786 - 1795. doi:10.1016/j.paid.2007.05.017

Valadez, M. de los D., Pérez, L. y Beltrán, J. (2010). La inteligencia emocional de los adolescentes talentosos. Faisca, 15(17), 2-17.

Valls, F. (2007). Inteligencia emocional y asesoramiento vocacional y profesional: usos y abusos. Electronic Journal of Research in Educational Psychology, 5(1), 179-200.

Woitaszewski, S.A. y Aalsma, M.C. (2004). The contribution of emotional intelligence to the social and academic success of gifted adolescents as measured by the Multifactor Emotional Intelligence Scale - Adolescent version. Roeper Review, 27(1), 25-30. doi: 10.1080/02783190409554285

Zavala, M.A., Valadez, M. de los D. y Vargas, M.C. (2008). Inteligencia emocional y habilidades sociales en adolescentes con alta aceptación social. Revista Electrónica de Investigación Psicoeducativa, 15(6), 319-338.

Zeidner, M., Shani-Zinovich, I y Matthews, G. (2005). Assessing emotional intelligence in gifted and non-gifted High school students: Outcomes depend on the measure. Intelligence, 33(4), 369-391. doi:10.1016/j.intell.2005.03.001 